\title{
REASSURANCE DU CUMUL D'ACCIDENTS *)
}

\author{
par \\ JEAN SOUSSELIER \\ Paris, France
}

Une Compagnie d'assurances délivre par l'intermédiaire d'Agences de Voyage des contrats d'assurances individuelles garantissant les personnes prenant place dans des autocars contre les décès par accident. Le capital garanti est uniforme. Il sera pris égal à I par simplification.

Cette Compagnie prend à sa charge le paiement de I décès au maximum dans chaque accident et demande à un Réassureur de payer, aux termes d'un traité en excédent de sinistre, les autres décès; autrement dit, si un accident cause $d$ décès, la Compagnie paie I, le Réassureur en excess paie $d-\mathrm{I}$.

Il s'agit de calculer la prime de ce traité en excédent de sinistre ? $\left.{ }^{1}\right)$

La solution dépend de trois paramètres:

$n$ nombre de passagers par autocar, égal au maximum au nombre de places; on supposera, pour fixer les idées, $n=5^{2}$ ).

$d$ nombre de décès survenus dans un même accident. C'est une variable stochastique comprise entre o et $n$.

Il n'y a pas lieu de faire intervenir la fréquence d'accidents (entraînant des morts) $q$, car celle-ci est un des éléments constitutifs du tarif appliqué par la Cédante et la prime de réassurance est exprimée comme une fraction de la prime originale. Si celle-ci est incorrecte, trop basse ou trop élevée, l'Assureur réalise une perte ou un bénéfice et il est normal que le Réassureur suive en cela la fortune de sa Cédante. La seule question est celle d'un

*) Rapport présenté au Colloque I96I à Rättvik (Suède)

1) On traiterait de même le problème plus général où, au lieu de I décès, la Compagnie d'assurances prendrait à sa charge $k$ décès.

2) Il faudrait tenir compte, en réalité, des variations de $n$ (loi de distribution de $n$ ) du fait que les autocars n'ont pas la même capacité et ne sont pas egalement remplis. 
partage équitable de la prime perçue, bonne ou mauvaise, entre la Cédante et le Réassureur.

$a$ nombre d'assurés par autocar; variable stochastique comprise entre o et $n$.

Il est normal que le nombre d'assurés, $a$, intervienne dans la fixation de la prime. $\mathrm{Si}$, en effet, un autocar accidenté ne contient qu'un assuré, le Réassureur n'aura rien à payer quel que soit le nombre des morts $d$. Si un autocar contient un nombre minime d'assurés - $a$ petit - il est improbable que le Réassureur ait à intervenir sauf si le nombre de victimes $d$ est considérable, auquel cas certaines de ces victimes seront des assurés. A la limite, si tous les passagers sont assurés, $a=n$, le Réassureur aura à payer $(d-\mathrm{I})$; il interviendra donc dès que $d>\mathrm{I}$.

D'une manière précise, il faut calculer la probabilité pour que dans un autocar comportant $n$ passagers dont $a$ sont assurés, impliqué dans un accident causant $d$ décès, il y en ait $x$ qui soient assurés, probabilité que nous noterons:

$$
P \stackrel{(a x)}{(n d)}
$$

c'est-à-dire $x$ assurés décédés parmi $d$ décès pour un autocar de $n$ personnes dont $a$ assurés $-0 \leqslant x \leqslant d$.

Il s'agit d'un problème simple d'analyse combinatoire. La formule est d'après les probabilités composées:

$$
\begin{aligned}
& P \underset{(n d)}{(a x)}=\left(\begin{array}{l}
d \\
x
\end{array}\right) \\
& \frac{a(a-\mathrm{I}) \ldots(a-x+\mathrm{I})(n-a) \ldots(n-a-d+x+\mathrm{I})}{n(n-\mathrm{I}) \ldots \ldots \ldots \ldots \ldots \ldots \ldots \ldots \ldots}
\end{aligned}
$$

en supposant toutefois qu'on ait à la fois $\begin{aligned} & (d \leqslant a \\ & (d \leqslant n-a\end{aligned}$

Si $d>a, x$ est au maximum égal à $a($ et non à $d)$ et la probabilité limite est:

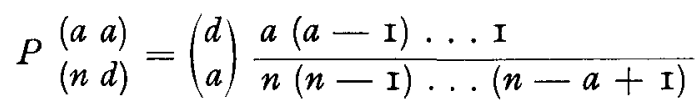

Si $d>(n-a)$, $x$ est au minimum égal à $(d-(n-a))$ et non 
à 0 ; il y a nécessairement des morts parmi les assurés. La formule ci-dessus est valable, en substituant au nombre des décès $d$ le nombre des survivants $s$, et au nombre $x$ des décès d'assurés, le nombre $y$ des survivants:

$$
\begin{aligned}
& d+s=n \\
& x+y=a
\end{aligned}
$$

Ayant calculé les probabilités $P$, on obtient la prime partielle d'excess $r_{d}$ par la formule:

$$
r_{d}=\Sigma_{x}(x-\mathrm{I}) \quad P \underset{(n d)}{(a x)}
$$

C'est la prime pure que doit recevoir le réassureur pour couvrir les $(x-\mathbf{I})$ sinistres d'assurés dans le cas où le nombre des décès est exactement $d$.

Pour chaque valeur de $a$ donnée, on obtient ainsi des valeurs de la prime d'excess $r_{d}$ croissant avec $d$, de o pour $d=\mathrm{I}$ jusqu'à $a-\mathrm{I}$ pour $d=5$.

La prime partielle d'assurance originale pour $d$ décès est de même

$$
p_{d}=\Sigma_{x} x \quad P \underset{(n d)}{(a x)}=\frac{a}{n} d
$$

Elle est en effet proportionnelle à la prime $d$ qui serait perçue si les $n$ passagers étaient assurés ${ }^{1}$ ).

Cette égalité permet une simplification du calcul de $r_{d}$-en remarquant que $\Sigma_{x} P \begin{aligned} & \left(\begin{array}{ll}a & x\end{array}\right) \\ & (n d)\end{aligned}=\mathrm{I}$

$$
p_{d}=r_{d}+\mathrm{I}-P\left(\begin{array}{ll}
(a & o
\end{array}\right)
$$

ce qui conduit pour: $a=2 r_{d}=\frac{d(d-\mathrm{I})}{n(n-\mathrm{I})}$ calcul facile

$$
a=3 \quad r_{d}=\frac{d(d-\mathrm{I})(3 n-4-d)}{n(n-\mathrm{I})(n-2)}
$$

et ainsi de suite; les calculs sont très laborieux.

$$
\text { Pour: } a=50 r_{d}=d-\mathbf{I}
$$

1) Il revient au même de dire qu'il y a $(n-a)$ passagers non assurés ou qu'il sont assurés auprès d'une autre Compagnie - ce qui implique le partage proportionnel de la prime. 
Ayant calculé les $r_{d}$ et $p_{d}$, il faut maintenant connaître la loi de distribution des sinistres $U_{d}$, c'est-à-dire la probabilité pour que dans un car accidenté comportant des morts, il y ait exactement $d$ décès $-\Sigma U_{d}=\mathrm{I}$.

C'est une certaine loi - loi de Poisson ou courbe en $U$ - statistiquement déterminable qui dépend du type d'autocars, du genre de voyages effectués etc....

Le taux de prime d'excédent de sinistre cherché est alors:

$$
\tau=\frac{\Sigma r_{d} U_{d}}{\Sigma p_{d} U_{d}}=\frac{\Sigma r_{d} U_{d}}{\frac{a}{n} \Sigma d U_{d}}
$$

Cette prime est fonction de la loi de distribution $U_{d}$ retenue, évidemment, mais elle est aussi fonction de $a$.

Elle est égale à zéro pour $a=\mathrm{I}$, et croît rapidement pour atteindre des valeurs élevées, supérieures à $0,5^{\circ}$ pour $a=5^{1}{ }^{1}$ ).

Pour le montrer, nous considérerons deux lois de distribution qui constituent, selon nous, deux limites extrêmes:

I loi (loi exponentielle) la probabilité de décès décroît en progression géométrique

$$
\begin{aligned}
& \text { d I } \quad 2 \quad 3 \ldots 5^{\circ} \\
& \begin{array}{llll}
U_{d} & \frac{\mathrm{I}}{2} & \frac{\mathrm{I}}{4} & \mathrm{I} \\
8 & \ldots & \mathrm{I} & \frac{\mathrm{I}}{2^{50}}
\end{array}
\end{aligned}
$$

II loi (équipartition) les chances de $d$ décès sont égales:

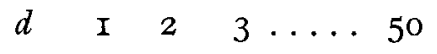

$$
\begin{aligned}
& U_{d} \quad \frac{\mathrm{I}}{50} \frac{\mathrm{I}}{50} \frac{\mathrm{I}}{50} \cdots \frac{\mathrm{I}}{50}
\end{aligned}
$$

Bien que la survenance de sinistres graves impliquant la mort de nombreux passagers ne soit pas exclue (autocar tombant dans un ravin, collision avec un train), il est certain que l'hypothèse d'équipartition des sinistres est tout-à-fait excessive: il y a une

1) C'est-à-dire plus de $50 \%$ de la prime pure; rapporté à la prime brute cela ferait moins, mais il faut en plus un chargement important pour tenir compte de la variance qui est considérable comme on peut le percevoir sans effort. 
large probabilité pour que les accidents ne causent qu'un petit nombre de morts. Néanmoins, la loi exponentielle va trop loin dans ce sens: la décroissance des probabilités est si rapide qu'elle exclut pratiquement tous les sinistres très importants.

Le problème que nous traitons pourrait se présenter identiquement pour l'assurance de passagers à bord d'avions. Dans ce cas, la loi de distribution des sinistres pourrait beaucoup plus se rapprocher de l'équipartition.

La figure I montre la variation rapide du taux de prime de réassurance en fonction du nombre de passagers assurés $a$.

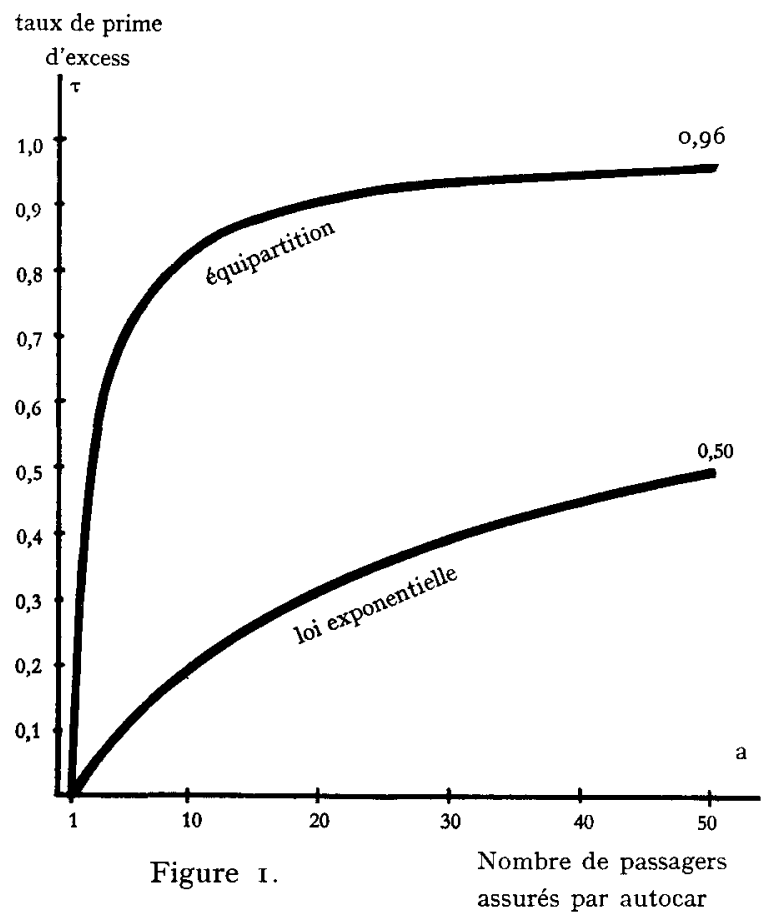

Pour la loi exponentielle on trouve:

$$
\begin{aligned}
& \text { pour } a=2 \tau=0,02 \\
& \text { pour } a=3 \tau=0,04 \\
& \text { pour } a=50 \quad \tau=0,50
\end{aligned}
$$


Ce dernier résultat se vérifie par un calcul direct:

$$
\begin{gathered}
p=q\left(\frac{\mathrm{I}}{2}+\frac{2}{4}+\frac{3}{8}+\frac{4}{\mathrm{I} 6} \ldots\right) \\
r=q\left(\frac{\mathrm{I}}{4}+\frac{2}{8}+\frac{3}{\mathrm{I} 6} \ldots\right) \\
\text { donc } r=\frac{p}{2}
\end{gathered}
$$

Pour la loi d'équipartition, on $a$ :

$$
\begin{aligned}
& a=2 \\
& \tau=\frac{\Sigma d(d-\mathrm{I}) / n(n-\mathrm{I})}{\frac{a}{n} \Sigma d}=\frac{\frac{n(n+\mathrm{I})(2 n+\mathrm{I})}{6}-\frac{n(n+\mathrm{I})}{2}}{\frac{2}{n} \frac{n(n+\mathrm{I})}{2} n(n-\mathrm{I})}=\frac{\mathrm{I}}{3} \\
& a=3 \quad \tau=\frac{\mathrm{I}}{2}
\end{aligned}
$$

D'une manière générale (5) $\tau=\frac{a-1}{a+\mathrm{I}}$ indépendant de $n^{1}$ ). Le taux ultime est:

$$
\frac{49}{5 \mathrm{I}}=0,96 \text { pour } a=5^{\circ}
$$

On peut admettre que c'est entre ces deux courbes extrêmes que se situe la progression effective des taux de prime cherchés.

La forte variation de taux de prime d'excess en fonction du nombre d'assurés, bien que prévue, est déconcertante.

1), Démonstration. Montrons d'abord que $\sum_{d} P \underset{(n d)}{(a)}=\frac{n-a}{a+\mathbf{I}}$

En effet, par récurrence, la proposition étant vraie pour $n=a+\mathrm{I}$ :

$\sum_{a} P\left(\begin{array}{ll}\left(\begin{array}{ll}n & o\end{array}\right) \\ (n)\end{array}\right)=\frac{n-a}{n}+\frac{(n-a)(n-a-\mathrm{I})}{n(n-\mathrm{I})}+\ldots=\frac{n-a}{n}\left(\mathrm{I}+\frac{n-a-\mathrm{I}}{a+\mathrm{I}}\right)=$ $=\frac{n-a}{a+\mathbf{I}}$

La formule (4) donne alors par sommation:

$$
\begin{gathered}
\Sigma p_{d}=\Sigma r_{d}+n-\Sigma P\left(\begin{array}{l}
(a \text { o }) \\
(n d)
\end{array}\right. \\
\text { d'où : } \tau=\frac{\Sigma r_{d}}{\Sigma p_{d}}=\frac{\frac{a}{n} \frac{n(n+\mathrm{I})}{2}-n+\frac{n-a}{a+\mathrm{I}}}{\frac{a}{n} \frac{n+(n+\mathrm{I})}{2}}=\frac{a-\mathrm{I}}{a+\mathrm{I}}
\end{gathered}
$$


En effet, rien ne permet de connaître à l'avance la proportion de passagers assurés. Elle dépendra entièrement du succès commercial de l'opération entreprise et variera avec le temps.

Rien ne permet donc d'assigner à la prime de réassurance une valeur acceptable. Paradoxalement, son taux devrait être d'autant plus élevé que le nombre d'assurés augmente: la loi des grands nombres joue ici en défaveur du réassureur.

\section{CUMUL D'ASSURÉS DANS DES VOITURES PARTICULIÈRES}

Il s'agit d'un problème dont la similitude avec celui traité ne doit pas faire illusion.

Une Compagnie d'assurances désire protéger son portefeuille d'assurances individuelles contre les dangers de cumuls du fait que plusieurs de ses assurés peuvent prendre place dans une même voiture particulière (4 places), éventuellement le même autocar. (Dans la pratique, le problème se complique du fait que les capitaux assurés ne sont pas les mêmes). Elle demande, comme ci-dessus, à un réassureur de la garantir en prenant en charge les sinistres excédant I décès.

Limitons-nous au cas où l'on considère seulement des voitures particulières à 4 places $(n=4)$ - On calculerait de même les taux de prime d'excess pour le cas des autres véhicules $n>4$ et la prime totale résulterait de la somme de ces différentes primes, en adoptant une loi de répartition convenable des différents véhicules.

Du point de vue mathématique, la solution est identique. Donnons ici le détail des calculs - élémentaires -.

Les deux lois de distribution de sinistres adoptées seront:

- l'équipartition: $\frac{I}{4}$ chance d'avoir I, 2, 3 ou 4 décès.

— une loi genre exponentielle: $\begin{array}{ccccc}d & \mathrm{I} & 2 & 3 & 4 \\ U_{d} & 0,50 & 0,25 & 0, \mathrm{I} 5 & 0, \mathrm{IO}\end{array}$

La prime d'assurance originale est alors:

- en équipartition: $\frac{\mathrm{I}}{4}(\mathrm{I}+2+3+4)=\frac{\text { IO }}{4}$ pour 4 assurés, c'est-à-dire $\frac{5}{4}$ pour $2, \frac{15}{8}$ pour 3 . 
- avec la loi $0,50-0,25-0, I_{5}-0$, IO : I $\times 0,5^{0}+2 \times 0,25$ $+3 \times 0, I 5+4 \times 0, I 0=\mathrm{I}, 85$ pour 4 assurés, c'est-à-dire 0,925 pour $2, I, 388$ pour 3 .

$a=2$

nombre de morts-assurés

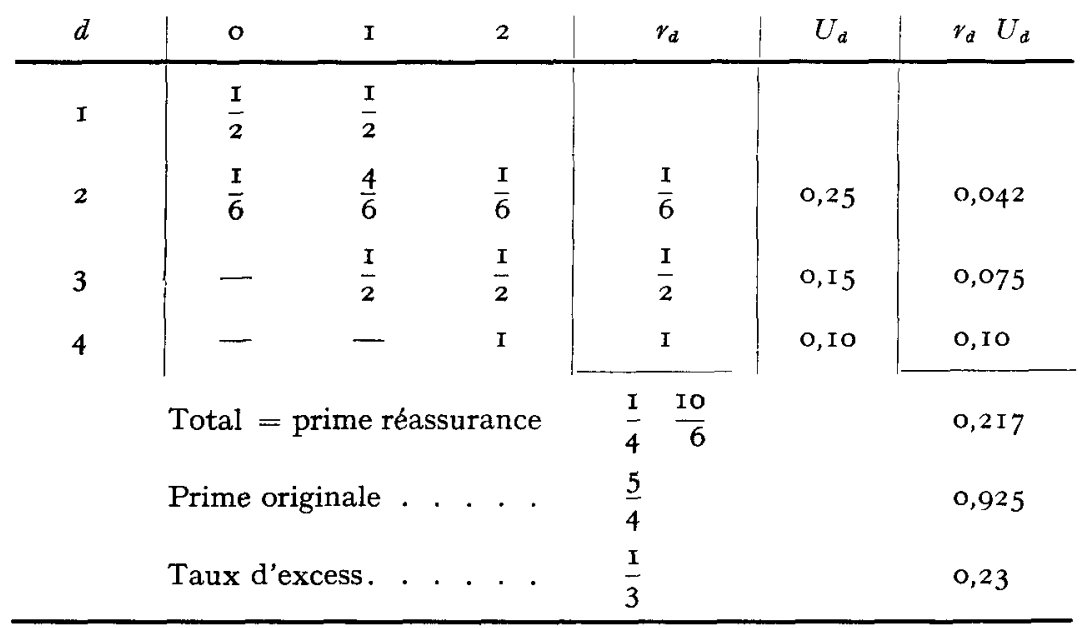

$a=3$

nombre de morts-assurés

\begin{tabular}{|c|c|c|c|c|c|c|c|c|}
\hline$d$ & o & $\mathbf{I}$ & 2 & 3 & & $v_{d}$ & $U_{d}$ & $v_{d} U_{d}$ \\
\hline $\mathbf{I}$ & $\frac{I}{4}$ & $\frac{3}{4}$ & & & & & & \\
\hline 2 & - & $\frac{I}{2}$ & $\frac{I}{2}$ & & & $\frac{\mathrm{I}}{2}$ & 0,25 & 0,125 \\
\hline 3 & - & - & $\frac{3}{4}$ & $\frac{I}{4}$ & & $\frac{5}{4}$ & 0,15 & $0, \mathrm{I} 875$ \\
\hline 4 & 一 & - & - & $\mathbf{I}$ & & 2 & O, IO & 0,20 \\
\hline \multicolumn{5}{|c|}{ Total $=$ prime réassurance } & $\frac{I}{4}$ & $\frac{\text { I } 5}{4}$ & & 0,512 \\
\hline & \multicolumn{3}{|c|}{ Prime originale } & . & $\underline{3}$ & $\frac{\text { IO }}{4}$ & & 1,388 \\
\hline & \multicolumn{2}{|c|}{ Taux d'excess } & . . & & $\frac{I}{2}$ & & & 0,37 \\
\hline
\end{tabular}




$$
a=4
$$

nombre de morts-assurés

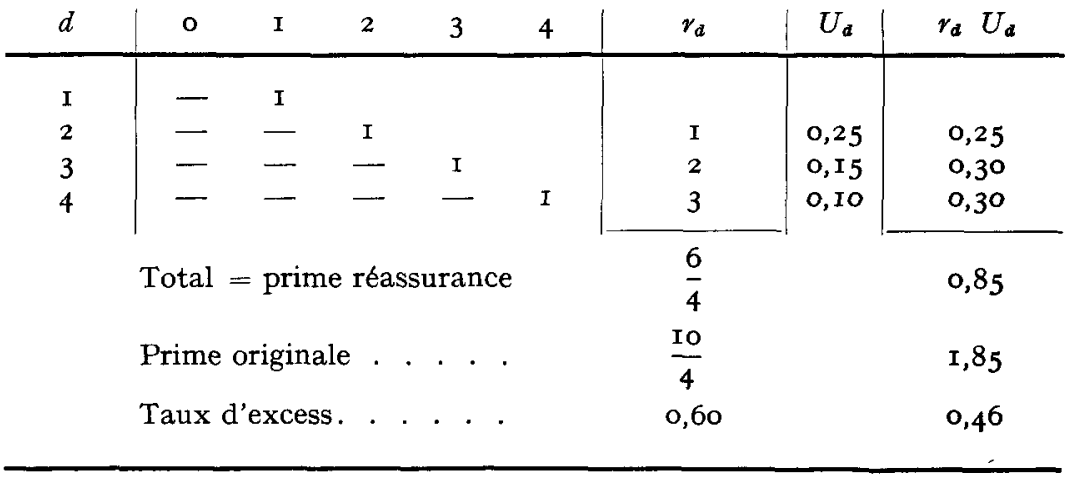

Avec l'équipartition on retrouve bien les taux obtenus précédemment (dans le cas de $n=5$ )

$$
\text { pour } \begin{array}{rlr}
a & =2 & 0,33 \\
a & =3 & 0,50 \\
a & =4 & 0,60
\end{array}
$$

conforme à la formule $\frac{a-I}{a+I}$

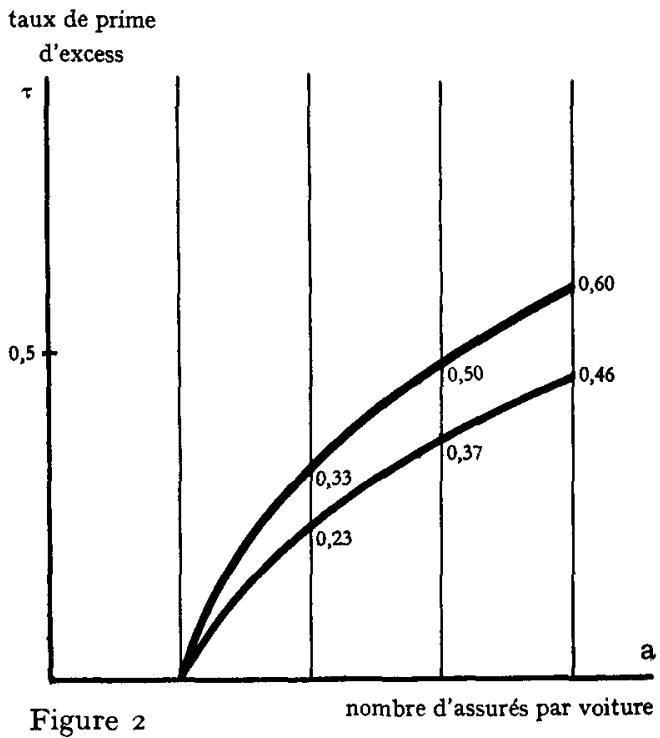


Avec la loi $0,25-0,15-0$, ro on a des taux d'excess plus admissibles: $\quad 0,23,0,37,0,46 \quad$ (Fig. 2).

Pour fixer un taux d'excess à la Compagnie, le Réassureur doit maintenant connaître la distribution des voitures selon la proportion des assurés occupants. Plus exactement, car cette distribution n'est pas forcément la même, la loi de distribution d'assurés dans les voitures accidentées (avec cas de morts). C'est ici que le raisonnement diffère:

Dans le cas des assurances délivrées en vue de voyages en autocars - et à l'occasion de ces voyages - la proportion $\frac{a}{n}$ d'assurés dépendait de la réussite commerciale de l'opération et était, par suite, indéterminable.

Ici, on est en présence d'une répartition statistique $\frac{a}{n}$, éventuellement déterminable par l'expérience et relativement constante dans le temps.

Si on admet, par exemple, qu'il y a $\frac{I}{\text { IO }}$ chances qu'il y ait deux assurés ensemble dans la même voiture, $\left.{ }^{1}\right) \frac{I}{5^{0}}$ qu'il y en ait trois, $\frac{I}{\text { IOO }}$ qu'il y en ait quatre, la prime d'excess sera ${ }^{2}$ ):

$$
\frac{\frac{2}{10} 0,23+\frac{3}{50} 0,37+\frac{4}{100} 0,46}{0,87+\frac{2}{10}+\frac{3}{50}+\frac{4}{100}}=0,074 \text { soit } 7,40 \%
$$

Ce pourcentage doit être appliqué à la fraction de la prime originale qui sert à couvrir les risques de décès à l'occasion de la circulation en voitures automobiles - mettons $50 \%$ -

Le taux d'excess est alors: $7,40 \% \times 0,50=3,70 \%$

On voit clairement que, malgré les apparences de similitude, le second problème n'est pas de même nature que le premier.

1) La présence de plusieurs assurés dans le même véhicule ne résulte pas seulement du hasard: il peut y avoir dans le portefeuille assuré une plus ou moins grande proportion d'assurés appartenant à la même famille ou à la même entreprise.

2) Par suite, 0,87 chance pour qu'il n'y ait qu'un assuré seulement. 